\title{
Color Content based Video Retrieval
}

\author{
Smita Chavan \\ Assistant Professor \\ Dept of Information Technology \\ Govt. College of Engineering \\ Aurangabad
}

\author{
Shubhangi Sapkal \\ Assistant Professor \\ Dept of Comp.Sci \& Engg \\ Govt. College of Engineering \\ Aurangabad
}

\begin{abstract}
Video retrieval is regarded as one of the most important in multimedia research. There are different types of representations for video i.e. low level representations and high level representations having different features based on the particular video annotating semantic concepts which appear in video shots is a challenging task. Integrated semantic based approach is proposed for similarity computations to improve the retrieval effectiveness in content based video retrieval. Video retrieval can be used for multiuser systems for video search and browsing which are useful in web applications. Here a DCT may be used that gives best performance among the different videos. We can introduce in this paper an interactive multiuser video retrieval framework composed by 1 . Service oriented architecturesystem which provides services for video semantic and semantic annotation, search and browsing for different domains 2. Web based interfaces for interactive query composition achieves browsing, annotation and visualization 3. A multitouch interface featuring gives collaborative natural interaction application. The performance of this technique is done with the help of cross over points of average precision and recall values of the data query videos fired on the whole database. The higher performance is achieved by HSV color space rather using RGB color space.
\end{abstract}

\section{General Terms}

Image Retrieval, Video retrieval

\section{Keywords}

Retrieval techniques, Color spaces, Similarity measure, Web based interface

\section{INTRODUCTION}

The need to query and process large amounts of data cannot be easily described as the video data. Content based methods are applied in order to improve in multimedia retreieval.To increase the effectiveness of content based methods can play essential roles which are used in media collections and enhance retrieval accuracy. The quality similarity measure employed for mapping textual query terms to visual concepts is considered as a key factor in content based retrieval. We can apply semantic similarity measure instead of measure. Interacting with multimedia data and video in particular requires more than connecting with data banks and delivering data via networks customer homes and offices. Macro level similarity measurement can be used to identify preliminaries for successful queries as the basis for the implementation of a query engine. Our goal is to investigate how content based video search can enhance the performance of traditional retrieval. Manual description of shots is not realistic machines may fill the gap with the automatic shot descriptions associated with the content based video retrieval. Searches might rely on metadata such as captions or keywords generated by human and store alongside each video in the database Color based retrieval is the backbone for content based video retrieval. The choice of color representations affects greatly to the efficiency of color based retrieval. Color based retrieval comparisons have been made with traditional color histograms in different color spaces to find efficient representations for the feature. Video indexing should be analogous to text document indexing where we perform a structural analysis to decompose a document into paragraphs, sentences and words, therefore content based video retrieval is developing technologies to automatically parse videos, audio and text to identify meaningful composition structure to extract and represent content attributes of any video resources.

\section{RELATED WORK}

\subsection{Content Based Video Retrieval}

CBVR has two approaches attribute based and object based Content Based Video Retrieval can be done by color, texture, shape, spatial relationship, semantic primitives, browsing, objective attribute, subjective attribute, motion text and domain concepts. By using content of videos as a feature vector and storing those processing of queries fired by user and comparing queries feature vector and feature vector of each video

Content Based Video Retrieval has two phases

\subsubsection{Database Population phase}

It has three main procedures

\subsubsection{Video Shot Boundary Detection}

Shot boundary detection can be implemented with partition segments. In digital video analysis, the longest sequence of frames that creates continuous, consistent movement within mechanical limitations of recording device is called a shot noted as Shot properties such as consistent motion flow contain mechanical information about the shot and will be utilized by analysis algorithm for video indexing purpose. The temporal changes of video shot color content can be described by using a temporal correlogram.Instead of color histograms temporal correlograms encapsulate the temporal changes of small spatial color relations.

\subsection{Key frame selection}

Key frame selection is based on selecting characteristics; key frames play an important role in video abstraction process. Key frames are still images extracted from original video data that best represent the content of the shots in an abstract manner. Often we use key frames to supplement the text of video $\log$. The representational power of a set of key frames depends on how they are chosen from all frames of a sequence. Not all video image frames within a sequence are equally descriptive and the challenge is how to automatically determine which frames are most representative and challenging task is to detect a hierarchical set of key frames such that a subset at a given level represents a certain granularity of video content which is critical for content based video browsing 


\subsection{Feature Extraction}

It is extracting low level spatial features like color, texture, shape. Structure layout and motion. An effective strategy in video content analysis is to use attributes extractable from multimedia resources. Much valuable information is also carried in other media components such as text superimposed on the images or included as closed captions. Characterizing video program for both consumer and professional applications

\subsubsection{Video Retrieval Phase}

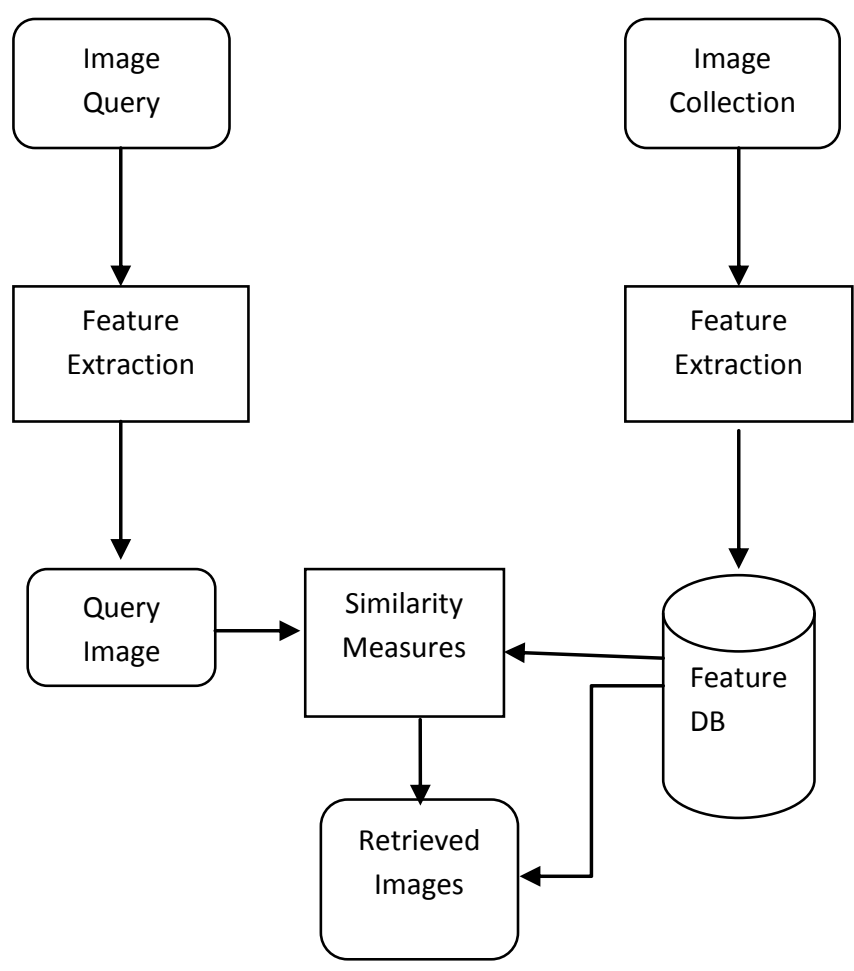

Fig 1: Content based video retrieval view

Absolute difference (AD) is used for feature vectors of the similarity measure based on different types are calculated with the help of Absolute difference (AD). In video retrieval based on content. Query video feature vectors are compared with all feature vectors which are stored in a database. Calculated differences will be ordered in increasing fashion so as to show result on top and which are relevant that are at lower level as per fig 1 view of CBVR.

\section{PROJECT GOALS}

Most simple database storage-description of videos as index along with the video. Human effort is involved in this case. We are searching for automotive search indexing and digital image storage method, i.e. latent semantic indexing (LSI).LSI is using vector space model which is low rank approximations of vector space represent image document collection. Original matrix is replaced by an as close as possible matrix where its columns space is only the subspace of the original matrix column space. By reducing the rank of the matrix noises (duplicate frames are reduces to improve storage and retrieval performance. Term indexing is referred to the process of assigning terms to the content of the videos. Closest terms in the database are returned based on the similarity measure between the query images and the resulting ones. Similarity measure is used in vector space model. Cosine similarity measure on term by video matrix vectors in a database. Oracle introduces new object type ORDimage which contains four different visual attributes global local color, texture and shape. ORDimage index provides multidimensional image structure to speed up stored feature vectors.

\section{METHODS \\ 4.1 Discrete Cosine Transform}

DCT is a separate linear transformation. It is two dimensional transform is equivalent to one dimensional DCT performed along a single dimension followed by one dimensional DCT in the other dimension. If we apply DCT to real data result is also real. DCT is a Fourier-related transform similar to the discrete Fourier transform (DFT), but using only real numbers. There are eight standard DCT variants, of which four are common. The most common variant of discrete cosine transform is the type-II DCT, which is often called simply the DCT its inverse, the type-III DCT, is correspondingly often called simply the inverse DCT" or the IDCT. Two related transforms are the discrete sine transforms (DST), which is equivalent to a DFT of real and odd functions, and the modified discrete cosine transforms (MDCT), which is based on a DCT of overlapping data. Each frame and a subset of DCT coefficients for each block where used as a vector representation.

\subsection{Color Spaces}

HSV color space is used with HSV Correlograms probability of finding identical colors An Autocorrelograms of image gives the probability of finding identical colors at distance $G$ and it provides significant computational benefits over the correlogram In this paper, term HSV Color Correlogram equals to autocorrelogram and with condition the HSV Color Histogram feature is Conversion of pixel colors from RGB to HSV color space for the color histogram, difference is measured between a query image and a database. Color histograms are used for the different color spaces. Let I be an $\mathrm{X} * \mathrm{Y}$ image composed of pixels $\mathrm{p}(\mathrm{x}, \mathrm{y})$, let $[\mathrm{c}]$ denote the set of $\mathrm{C}$ color $\mathrm{C}, \mathrm{Cc}$ that can occur in the image and [D] denote a set of $\mathrm{D}$ fixed distances $\mathrm{d}$... $\mathrm{dD}$ which are measured using the $\mathrm{L} \infty$ norm. For a pixel P, let I (p) denote its color $\mathrm{C}$ and let Ic corresponds to a pixel $\mathrm{P}$, for which $\mathrm{I}(\mathrm{P})=\mathrm{C}$, the histogram

$\mathrm{h}(\mathrm{I})$ for color $\mathrm{Ci}$ and color pair $(\mathrm{Ci}, \mathrm{Cj})$ respectively as Where above description corresponds to a probability of any pixel in I being of color Ci.Five different quantization of the color space where evaluated with following hue, saturation and value combinations. An autocorelogram of the image I give the probability of finding identical colors $(\mathrm{Ci}=\mathrm{Cj})$ at distance $\mathrm{d}$ and it provides significant computational benefits over the correlograms. HSV color histogram is calculated as conversion of pixel colors from RGB to HSV color space.HSV color correlogram gives improved retrieval over RGB autocorrelogram.HSV color space is best over the other traditional color based methods.

\subsection{Performance Comparison}

Precision and recall are the two functions of the desired concept of the detectors performance specified by $\mathrm{p}$ and $\mathrm{q}$ of the user interval as $\mathrm{U}=[\mathrm{U}$ low\%, up\%] these are known as statistical performance comparison parameters. The performance of the retrieval system can be measured in terms of its recall and precision. Recall measure the ability of the system to retrieve all the models that are relevant, while precision where a represent the number of relevant images that are retrieved, B, the number of irrelevant items and the C, 
number of relevant items those where not retrieved. The number of relevant items retrieved is the number of the returned images that are similar to the query image the number of relevant items in collection is the number of images that are in the same particular category with the query image. The total number of items retrieved is the number of the images that are returned by the search engine

Definitions of perforamnce3parameters are as follows:

Precision $=$ Number of relevant videos retrieved $/$ Total number of videos retrieved $=\mathrm{A} / \mathrm{A}+\mathrm{B}$

Recall = Number of relevant videos retrieved $/$ Total number of relevant videos in database $=\mathrm{A} / \mathrm{A}+\mathrm{C}$

The goal is to jointly maximize precision and recall

Steps to implement:

In the implementation phase video may be TV video, natural images or collection of frames. With the use of DCT, similarity measure and HSV color space we will write an algorithm in two parts. First part will consist of Database creation with the help of different videos and samples. It will simulate to video file reading and then save this file into database. Search is on the basis of images. It can select images which are closest to the video. Simply first module will simple as just read video and create a database. Second module will be feature extraction and save it into database. Third module will image selection and comparison of the stored images along the databases

Platform will be Matlab with windows 7 .

\subsection{Experimental Results}

HSV color space and AD similarity measurements are considered for calculating the precision recall crossover points of proposed CBVR. Higher the crossover points value is better for the CBVR performance. Basic need is to handle fast growing database as well as efficiency of retrieval. The feature vectors are used for processing of query fired by user and comparing the queries feature vector and feature vector of each video. The relevant or closest matches are displayed as relevant results.

\section{FUTURE WORK}

\subsection{Audiovisual Archieve Retrieval}

Content Based Video Retrieval is maturing to the point where it can be used in real world retrieval practices. One such a practice is audiovisual archive whose users increasingly require fine grained access to broadcast television content. Its video retrieval community will be described with transcript based search low level feature based search and detector based search. Content based analysis and video retrieval methods have been evaluated extensively in TRECVID.Evaluation

Methodology used to evaluate the potential impact of CBVR in audiovisual archive.

\subsection{Interactive Multiuser Video Retrieval}

\subsubsection{Semantic Annotations}

It is based on the bag of words approach (BoW).it treats image or key frames as the visual analog of a document i.e. represented by a bag of quantized descriptors Ex. SIFT referred to as visual words. It gives spatial layout of the features

\subsubsection{Web Based User Interface}

A web based video search system with video streaming delivery to search videos obtained from PowerPoint presentations using the associated metadata. A mobile video browsing and retrieval applications based on HTML and JavaScript gives an interactive video browsing tool for supporting content management and selection in post production has been presented, comparing the usability of full featured desktop applications and with a limited web based interface.

\subsubsection{Multitouch Interface for Multimedia \\ Retrieval}

In this method one shared display which provides group members with a shared context and focus of attention, supporting collocated cooperative work known as single display groupware SPG

\subsection{Semantic Based Approach}

\subsubsection{Semantic Similarity Measures}

Semantic similarity measures are also called as text semantic measures. The similarity measure is used for computing the confidence score shows the degree of the relevancy between query and the shot, therefore for each query the confidence score of all shots should be computed. Two prominent works in video retrieval which utilized semantic similarity of words. There are two types of similarity measures, knowledge based and corpus based. Both the types utilized through trained concept detector in an unsupervised manner in order to solve video retrieval problem by employing concepts from natural language understanding. By using semantic word similarity the authors tried to amp query terms which stated in natural language with visual concepts. Knowledge based semantic word similarity measure is based on word Net, some query terms could not be mapped due to the lack of information content(IC)

\subsubsection{Information Retrieval}

To meet the different characteristics of information and coming up with better retrieval effectiveness are two

Significant factors which contribute to the construction of a

retrieval system. Various models in information retrieval try to enhance the effectiveness of text documents retrieval. Most common models for information retrieval are as follows

\subsubsection{Boolean Retrieval Model}

Boolean retrieval model is based on logical operators as "and" or"not" in which query parameters and database attributes are exactly matched.

\subsubsection{Vector Space Retrieval Model}

In Vector space retrieval model nonbinary weights are assigned to the index of documents and terms. So the similarity between index term and query is calculated by these binary weights.

\subsubsection{Latent Semantic Indexing Retrieval Model}

In Latent semantic indexing retrieval model a term correlation matrix is made by TF-IDF weights and then singular value decomposition is applied to the index term matrix for measuring similarity. In this model the query as a pseudo document should be modeled and the most similar documents in the projected concepts space should be found. 
Some information retrieval models based on fuzzy sets.fuzy information retrieval model is relevance of the document is defined as a degree of membership and the query is modeled as a fuzzy set of terms.

\subsubsection{Content Based Multimedia Information \\ Retrieval}

Multimedia combination of text, audio, animations, images, video images can occur in Content based multimedia information retrieval. Automatic video retrieval can be done based on query class model and the effectiveness of this model has been shown by experiments. Query classified into one of the category named as person, object, general object, scheme and sports. Video retrieval method text matching, ontology querying and semantic visual query are applied to select a relevant detector among a set of machine learned concept detectors

\section{CONCLUSION}

This paper focuses mainly on how Content Based Video Retrieval has new techniques as it can be used with different phases and also we can make it as a web based for the search engine experiments. Content based video retrieval can be implemented with the different similarity measures with its types using different color spaces specifically with the other color spaces. Performance of Content based video retrieval can be increased with the audiovisual archive retrieval and interactive multiuser video retrieval based on different similarity measures as absolute differences as shown in the Fig-2.According to our stored database output video can be obtained.

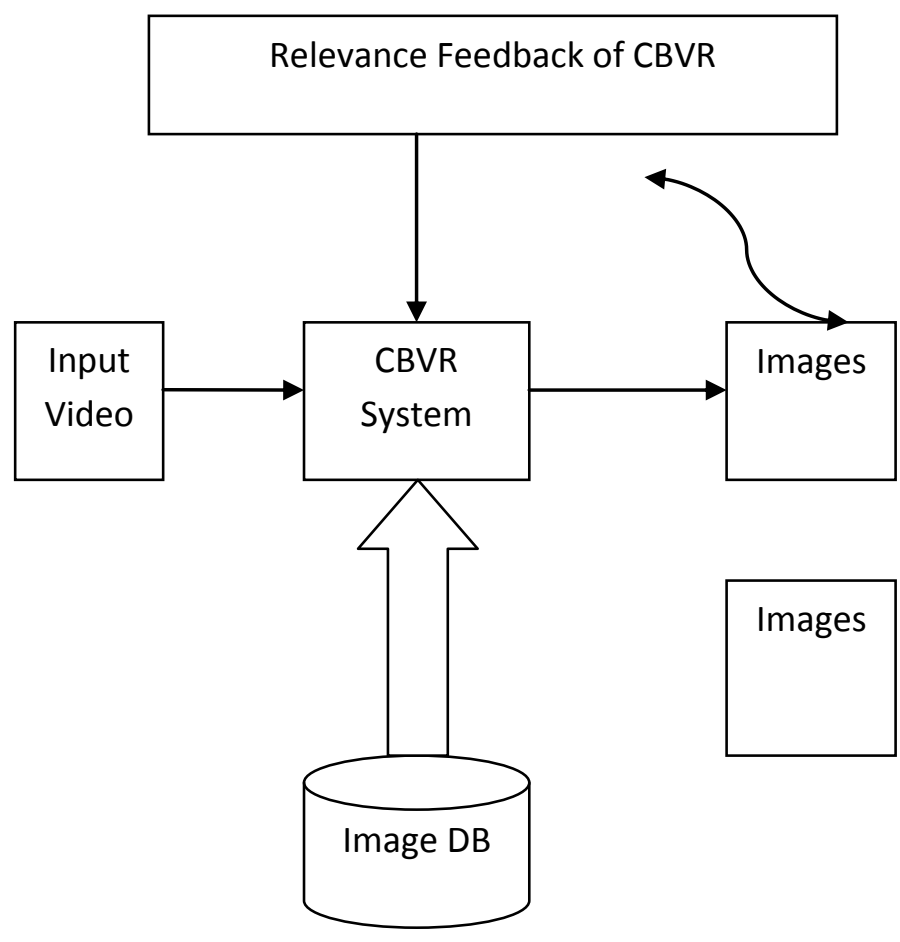

7. REFERENCES

[1] T. Welsh, M. Ashikhmin and K.Mueller, Transferring color to grayscale image, Proc. ACM SIGGRAPH 2002 vol.20, no.3, pp.277-280, 2002.

[2] Kekre, H. B., et al. "Improved Face Recognition with Multilevel BTC using Kekre's LUV Color Space." International Journal 3 (2012).

[3] H.B.Kekre, Sudeep D. Thepade, Sanchit Khandelwal, Karan Dhamejani, Adnan Azmi, "Face Recognition using Multilevel Block Truncation Coding" International Journal of Computer Applications (IJCA) December 2011 Edition.

[4] Aytar Y, Shah M, Luo J (2008) Utilizing semantic word similarity measures for video retrieval.In: IEEE conference on computer vision and pattern recognition. Anchorage, Alaska

[5] Bach J, Fuller C, Gupta A, Hampapur A, Horowitz B, Humphrey R, Jain R, Shu C, Sethi I, Jain R (1996) Virage image search engine: an open framework for image management. In: Storage and retrieval for stil image and video databases IV, vol 2670. SPIE, San Jose, CA, USA, p 87, 76

[6] Blair DC (1979) Information retrieval, 2nd edn. J Am 6:374-375. Soc Inf Sci 30doi:10.1002/asi.463030062

[7] Amant RS, Healey CG (2001) Usability guidelines for interactive search in direct manipulation systems. In: Proc of international joint conference on artificial intelligence, vol 2, pp 1179-1184

[8] Behmo R, Paragios N, Prinet V (2008) Graph commute times for image representation. In: Proc. of IEEE conference on computer vision and pattern recognition (CVPR), conference on image and video retrieval (CIVR), pp 133-142. Tempe, AZ, USA pp 1-8

[9] Bertini M, Del Bimbo A, Nunziati W (2006) Video clip matching using MPEG-7 descriptors and edit distance.

Fig 2: Feedback of CBVR 